\title{
Analysis of LFM-waveform Libraries for Cognitive Tracking Maneuvering Targets
}

\author{
Wang Hongyan ${ }^{1}$, Yu Daobin ${ }^{2}$ and Wu Yanhong ${ }^{2}$ \\ ${ }^{1}$ The Equipment Academy, The Information Equipment Department \&\& Science and Technology on Complex Electronic System Simulation \\ Laboratory, Beijing, China \\ ${ }^{2}$ The Equipment Academy, The Optoelectronic Equipment Department \&\& Science and Technology on Complex Electronic System \\ Simulation LaboratoryBeijing, China
}

\begin{abstract}
Based on the idea of the waveform agility in cognitive radars, the waveform libraries for maneuvering target tracking are discussed. LFM-waveform libraries are designed according to different combinations of chirp parameters and FrFT rotation angles. By applying the interact multiple model (IMM) algorithm in tracking maneuvering targets, transmitted waveform is called real time from the LFM-waveform libraries. The waveforms are selected from the library according to the criterion of maximum mutual information between the current state of knowledge of the model and the measurement. Simulation results show that waveform library containing certain amount LFM-waveforms can improve the performance of cognitive tracking radar.
\end{abstract}

\section{Introduction}

Cognitive radar can select transmitter configuration according to characteristics of the targets and environment and can improve the performance of detection and tracking [1]. It is adaptively, intelligently and dynamically.

The cognitive radar is proposed by Simon Haykin in 2006[2]. The components of the cognitive radar are discussed in his correlative papers [3-6]. The tracking filter and transmitter of the cognitive radar are regarded as a closed-loop system with the environment. The transmitted waveform is selected in real time to track targets effectively by taking advantage of all available information. Study on optimal waveform design and scheduling based on signal-to-noise ratio (SNR) maximisation or information theory is preferred for cognitive radar. The optimization is too complex to obtain analytical solution and also have large amount of calculation. Probably the transmitter cannot tune into the optimal waveform in the observation period.

We have taken an information theoretic waveform optimization, which choose optimal waveform in several observation times for global optimization [7-9]. It is the problem of waveform scheduling. Targets tracking based on the waveform library Theory on design waveform library is introduced by some scholars [10-15], which is practical and operational for waveform agility in radars systems. It has a finite library of waveforms in the tracking system. An optimal choice is made based on the certain consideration for next transmitted waveform. The basic target tracking system with waveform library is shown in figure 1.

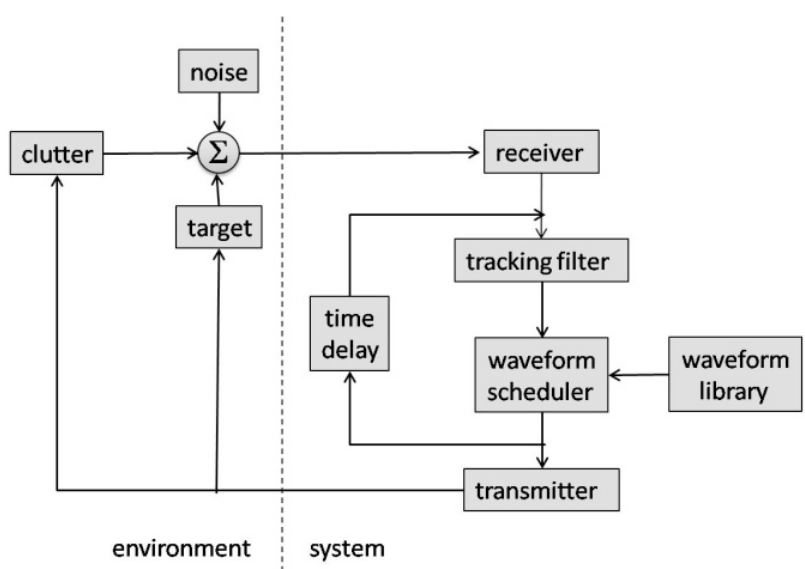

Figure 1. Target tracking system with waveform library

The idea of selecting waveforms simply based on the four different types according to their ambiguity functions was introduced in the paper of A. W. Rihaczek [10]. Work has been done in the area of waveform libraries design and scheduling algorithm by Cochran [11]. The design of waveform libraries was discussed using the informationtheoretic measures of effectiveness and the results of simulations comparing one-step ahead and multi-step ahead scheduling with an unscheduled approach are summarized. In the paper of Savage, waveform library was obtained by implementing a fractional Fourier transform (FrFT) for tracking accelerating targets within an interacting multiple model (IMM) framework [12]. A 
waveform selection approach for tracking the maneuver target described as polynomial prediction model is proposed by Tan. FrFT is also used to rotate the measurement error ellipse to select the optimal waveform [13]. The papers of Suvorova were discussing waveform library design and waveform selection criteria for target tracking by [14-15]. With this criterion the LFM and LFM-rotation waveform libraries need only the maximum and the minimum of chirp rate.

We focus on the design of LFM-waveform library for maneuvering target tracking. The IMM is introduced for the maneuvering targets of multiple possible dynamical models. Simulations are performed to validate the design of library and information-theoretic criteria for adaptive selection of a waveform.

\section{Problem Formulation}

The baseband signal is $s(t)$ and he transmitted pulse signal, $s_{T}(t)$, is [13]

$$
s_{T}(t)=\sqrt{2} \operatorname{Re}\left\{\sqrt{E_{T}} s(t) e^{j \omega_{c} t}\right\}
$$

where $\omega_{c}$ is carrier frequency, $E_{T}$ is emitted energy, $\operatorname{Re}\{\cdot\}$ denotes the real part. The received signal is

$$
s_{R}(t)=\sqrt{2} \operatorname{Re}\left\{\left[\sqrt{E_{R}} e^{j \phi} s(t-\tau) e^{j v t} \quad n(t)\right] e^{j \omega_{c} t}\right\}
$$

where $\phi$ is stochastic phase, $E_{R}$ is received energy, $n(t)$ is white Gaussian noise. $\tau$ is the time delay and $v$ is the Doppler shift. Range and the radial velocity of target can be described by the following equation:

$$
r=\frac{c \tau}{2}, \&=\frac{c v}{2 \omega_{c}}
$$

The salient transform of the down conversion of $S_{R}(t)$ is its ambiguity function $[12,13]$ :

$$
A(\tau, v)=\int_{-\infty}^{\infty} s\left(t-\frac{\tau}{2}\right) s^{*}\left(t+\frac{\tau}{2}\right) e^{-j \omega t} d t
$$

The ambiguity function is a two-dimensional function of time delay and Doppler shift. The maximum of $A(\tau, v)$ is at $(\tau, v)$, which is the measurement of time delay and Doppler shift under ideal conditions. Then range $r$ and velocity \& can be calculated by equation 3 .

The measurement noise covariance matrix $N\left(\theta_{k}\right)$ is waveform dependent:

$$
N\left(\theta_{k}\right)=T J^{-1} T^{T}
$$

where $\theta_{k}$ is the vector of waveform parameters, $J$ is the Fisher information matrix corresponding to the $\tau$ and $v$, $J^{-1}$ is the Cramer-Rao lower bound (CRLB) of the unbiased estimator of range and Doppler of a target as $A(\tau, v)$ at its maximum point.

The interacting multiple model (IMM) Kalman filter is approximate to optimal filter for linear dynamic model with Gaussian noise. It is not for the maneuvering target tracking. The interacting multiple model (IMM) filter is adaptive for the target with deceleration, acceleration or swerve dynamics. clutter and noise are not taken into account in the discus [12].
The IMM has structure as figure 2. It is the weighted summation to estimations of each filter with different tracking model. We assume that changes in target trajectory can be modelled as $n$ dynamic models, that is $m_{1}, m_{2}, \mathrm{~L}, m_{n}$. The state equation and measurement equation of dynamic model $m_{i}$ are

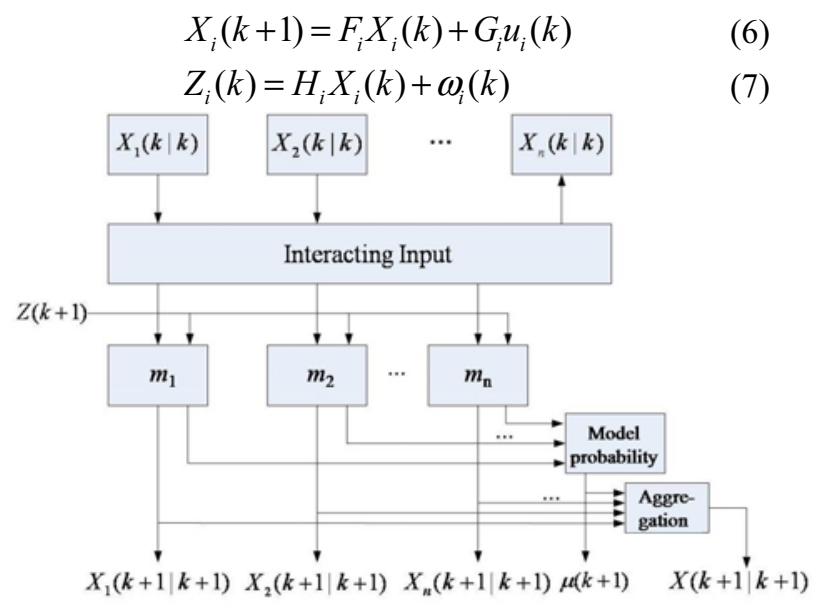

Figure 2. Diagram of IMM

where $i=1, \mathrm{~L}, n$ is the dynamic model at time. We write $X_{i}(k)$ for the state of the track and $Z_{i}(k)$ for the measurement at time $k . F_{i}$ is the state propagation matrix, $H_{i}$ is the measurement matrix and $G_{i}$ is Process noise matrix. Process noise is denoted by $u$ and the measurement noise by $\omega$. These both are zero mean, white, and uncorrelated Gaussian noise sequences with covariance matrix $Q_{i}(k)$ and $N(\theta)$ respectively.

Let $\mu_{i}(k-1 \mid k-1)$ denotes the initial probability of dynamic model $i$ and $M_{k}$ denotes the model set at time $k$. We assume the transition probabilities of dynamic models as $\pi_{i j}, i, j \in 1, \mathrm{~L}, n$. The algorithm of IMM is described as follows.

\subsection{Initialization}

The predictive model probability is

$$
\mu_{j}(k \mid k-1)=\sum_{m_{j} \in M_{k-1}} \pi_{i j}{ }_{i}\left(\begin{array}{ll}
k & 1
\end{array}\right)
$$

The initial conditional weight is

$$
\mu_{i j}=\pi_{i j}{ }_{i}(k-1) /{ }_{j}(k \mid k-1)
$$

The initial state estimate is calculated using

$$
X_{j}^{0}=\sum_{m_{j} \in M_{k-1}} X_{i}(k-1) \mu_{i j}
$$

and covariance is

$$
\begin{gathered}
P_{j}^{0}=\sum_{m_{j} \in M_{k}}\left[P_{j}(k-1 \mid k-1) \quad\left(X_{j}\left(\begin{array}{lll}
k & 1 \mid k & 1
\end{array}\right) \quad X_{j}^{0}\right)\right. \\
\left.\cdot\left(X_{j}(k-1 \mid k-1)-X_{j}^{0}\right)^{T}\right] \mu_{j}(k-1)
\end{gathered}
$$

$X_{i}(k-1 \mid k-1)$ denotes the output of $i$ th filter at time $k-1$.

\subsection{Estimation}


Each filters then use $X_{j}^{0}$ and $P_{j}^{0}$ as update of the state estimate and error covariance and calculate according to its model.

\subsection{Update model probability}

We assume the likelihood function and the probability of model as follows

$$
\begin{array}{r}
L_{j}(k)=N\left[\mathscr{Z} /(k) ; O, S_{j}\right] \\
\mu_{j}(k)=\frac{\mu_{j}(k \mid k-1) L_{j}(k)}{\sum_{m_{j} \in M_{k}} \mu_{i}(k-1) L_{i}(k)}
\end{array}
$$

where $\frac{Z j}{j}(k)$ is the innovation and $S_{j}$ is its covariance matrix. $N[\cdot]$ denotes normal distribution.

\subsection{Aggregation}

Aggregate state estimate and covariance information are given by

$$
\begin{gathered}
X(k \mid k)=\sum_{m_{j} \in M_{k}} X_{j}(k) \mu_{j}(k) \\
P(k \mid k)=\sum_{m_{j} \in M_{k}}\left[P_{j}(k \mid k)+\left(X_{j}(k \mid k) \quad X(k)\right)\right. \\
\left.\left(X_{j}(k \mid k)-X(k)\right)^{T}\right] \mu_{j}(k)
\end{gathered}
$$

The initial input will be the measurement $z_{0}$ or the parameters $\theta_{0}$ of waveform. Our motivation is to select optimal waveform of transmitter within the cognitive loop. Then we select $z_{0}$ as the initial input.

\section{LFM waveform library}

\subsection{Waveform library design}

The goal of waveform library design is to produce a waveform library that is limited, yet sufficient to provide a choice of high performance waveform.

When the measurement error ellipse is orthogonality with the one of the predicted estimated states, the waveform is the optimal for purpose of target tracking [16]. The measurement covariance matrix is dependent on the waveform. It is a selection of a waveform with certain measurement error. Within IMM framework, waveform library for each model consists of arbitrary transforms of a single waveform.

We describe the linear frequency modulation (LFM) or "chirp" waveform library. In this case the waveform is $s(t)=s_{0}(t) \exp \left(j b t^{2}\right)$. b is the chirp rate supported by the radar. The waveform library is constructed of the transforms with different rotation angles of LFM waveform. It is conducted by the fractional Fourier transform.

For this library the corresponding measurement covariance matrices $N_{\theta_{i}}$ are given by the equation

$$
\left\{\begin{array}{l}
N_{\theta_{i}}=S^{-1} N_{\theta_{0}} S \\
S=S(\varphi) * S(b)
\end{array}\right.
$$

where $\varphi$ is the rotation angle and $|S|=1$.

$$
S(\varphi)=\left[\begin{array}{cc}
\cos \varphi & -\sin \varphi \\
\sin \varphi & \cos \varphi
\end{array}\right], S(b)=\left[\begin{array}{cc}
1 & 0 \\
2 b & 1
\end{array}\right]
$$

\subsection{Waveform selection criteria}

For the adaptive selection, we would like to select the waveform, while the uncertainty of the dynamic model of the target is minimized at each echo. If we take a single step ahead approach, the waveform is the one, which minimizes the expected entropy of the probability distribution of the model at the next epoch. This implies that the mutual information between the model and the measurement needs to be maximized [13].

By the criterion of maximum mutual information between the current state of knowledge of the model and the measurement, we select the waveform from the library satisfying

$$
\begin{aligned}
& \theta_{o p t}=\underset{\theta \in\left\{\theta_{1}, \theta_{2}, \ldots, \theta_{n}\right\}}{\arg \min }\left(-\sum_{j=1}^{n} P_{j}(k \mid k-1) \log \frac{\left|N_{j, \theta}\right|}{\sum_{l=1}^{n} P_{l}(k \mid k-1) \log N_{l, \theta}}\right) \\
& \text { where the } P_{j}(k \mid k-1) \text { are the predictive model } \\
& \text { probabilities. }
\end{aligned}
$$

\section{Simulations}

The simulations were performed to validate the ideas above. There are multiple maneuvers for the target trajectories in range and Doppler, which are described in figure 3

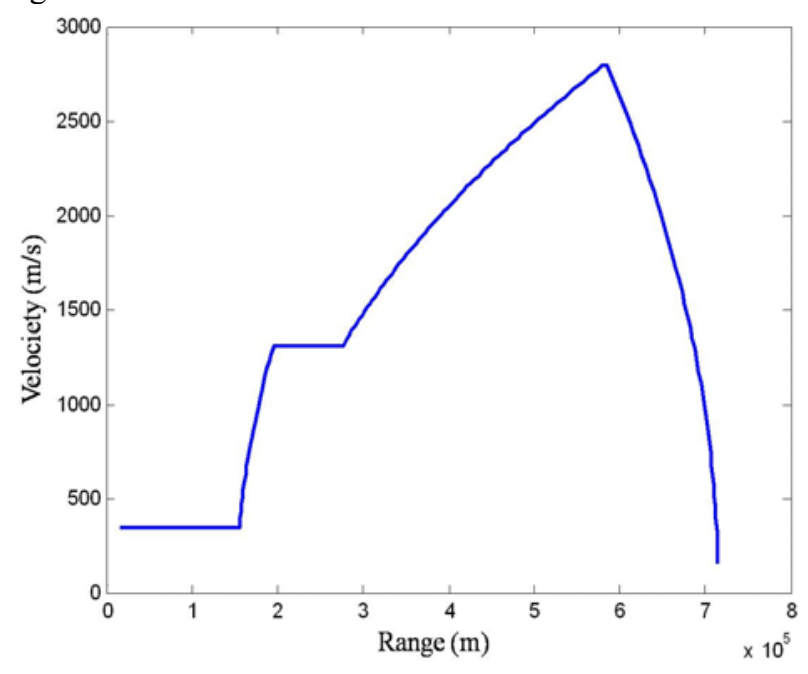

Figure 3. Manoeuvres for the target trajectory

The measurement interval is $\Delta t=2$. The simulation was run with the following parameters:

$$
F=\left[\begin{array}{cc}
1 & \Delta t \\
0 & 1
\end{array}\right], H=\left[\begin{array}{ll}
1 & 0 \\
0 & 1
\end{array}\right], G=\left[\begin{array}{l}
0 \\
1
\end{array}\right]
$$

The accelerated velocity of each dynamic model is $a_{i}=50,20,0,-20,-50, i \quad 1,2 \overline{\bar{\prime}} . ., 5$. The initial probability of each dynamic model is 0.2 . The model transition probability matrix of the Markov chain is 


$$
\Pi=\left[\begin{array}{lllll}
0.6 & 0.1 & 0.1 & 0.1 & 0.1 \\
0.1 & 0.6 & 0.1 & 0.1 & 0.1 \\
0.1 & 0.1 & 0.6 & 0.1 & 0.1 \\
0.1 & 0.1 & 0.1 & 0.6 & 0.1 \\
0.1 & 0.1 & 0.1 & 0.1 & 0.6
\end{array}\right]
$$

It is assumed that $N(0)=\left[\begin{array}{ll}\sigma_{1}^{2} & 0 \\ 0 & \sigma_{2}^{2}\end{array}\right]$. The range and rangerate standard deviations are $\sigma_{1}=10$ and $\sigma_{2}=1$ 。

In consider of the bandwidth of the transmitter, the chirp rate is limited. We define the waveform library $L_{1}$ containing one waveform $\left(b_{1}=b_{\max }\right)$ and $L_{2}$ containing two waveforms $\left(b_{1}=b_{\max }, b_{2}=b_{\min }\right)$. Based on the above considerations, we also define the waveform libraries containing six waveforms and ten waveforms.

$$
\begin{aligned}
& L_{6}=\left\{b_{1},\left(b_{1}, \phi=0.2 \pi\right),\left(b_{1}, \phi \quad 0.4 \pi\right), b_{2},\right. \\
& \left.\left(b_{2}, \phi=0.2 \pi\right),\left(b_{2}, \phi=0.4 \pi\right)\right\} \\
& L_{10}=\left\{b_{1},\left(b_{1}, \phi=0.1 \pi\right),\left(b_{1}, \phi \quad 0.2 \pi\right),\left(b_{1}, \phi \quad-0.3 \pi\right),\right. \\
& \left(b_{1}, \phi=-0.4 \pi\right), b_{2},\left(b_{2}, \phi=0.1 \pi\right),\left(b_{2}, \phi \quad 0.2 \pi\right), \\
& \left.\left(b_{2}, \phi=0.3 \pi\right),\left(b_{2}, \phi=0.4 \pi\right)\right\}
\end{aligned}
$$

The Monte Carlo simulations were performed 100 times. The accumulative error of the filter is given by

$$
e_{s}(k)=e_{s}(k-1) \quad \frac{1}{M} \sum_{i=1}^{M}\left[X_{i}(k) \quad X_{i}(k / k)\right]
$$

The choice of the optimal waveform from the LFM waveform library and the accumulative estimation errors are simulated with different initial estimation covariance. The different initial estimation covariance is taken into account in simulation.

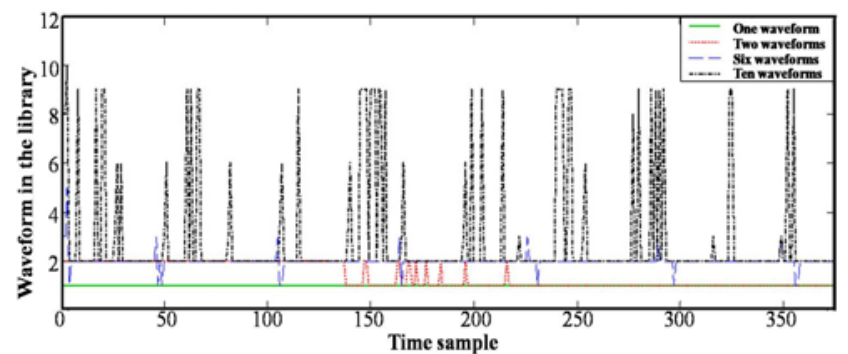

(a)

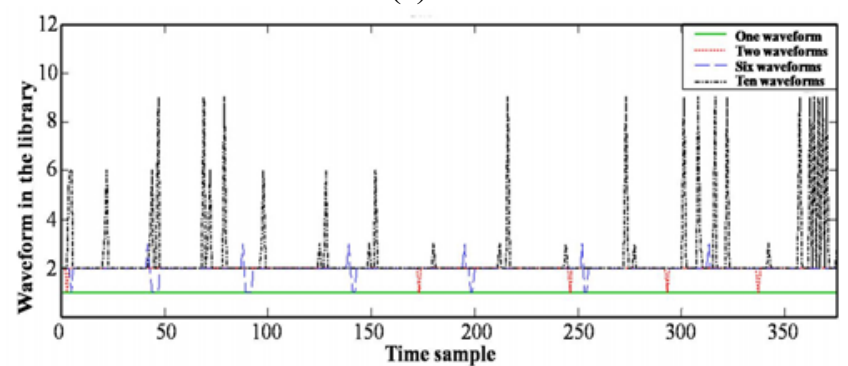

(b)

Figure 4. Waveform scheduling with different initial estimation covariance
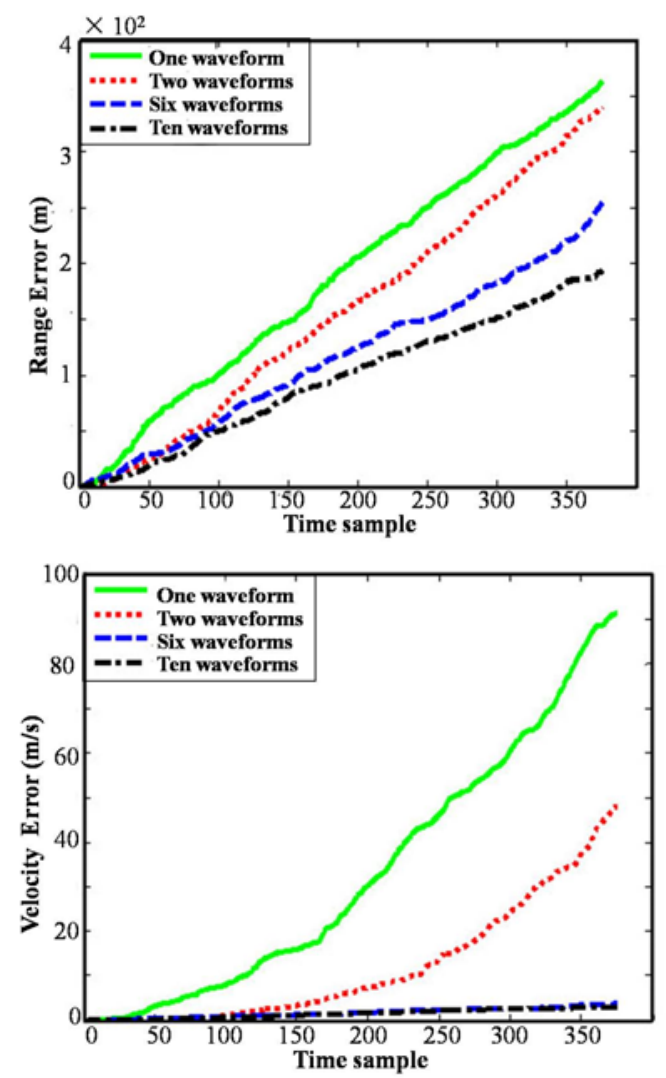

(a)
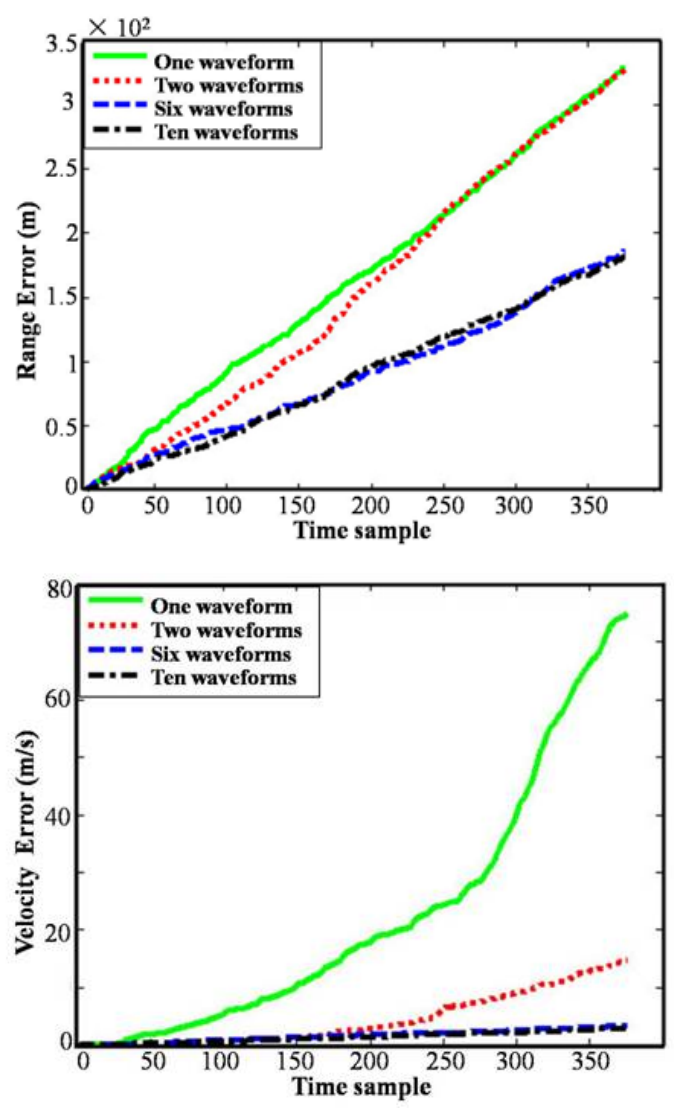

(b)

Figure 5. Accumulative errors in range and velocity for waveform selection with different initial estimation covariance 
The initial estimation covariance for results, shown in Figure 4(a) and Figure 5(a), is larger than the other. The curves in fugre 4 illustrate that, if the initial estimation covariance is larger, the waveform agility is frequently. The waveform scheduling is stably with the estimation covariance decreased. The waveform scheduling is related with the trajectories.

The range errors of results using $L_{1}$ and $L_{2}$ are similar, while the velocity error is better for library $L_{2}$. For the accelerated target in the distance, the choice of the waveform is frequently to maintain the velocity tracking error, but the range tracking error is deteriorated. The waveform library containing more waveforms is not necessarily effective to improve tracking performance.

The tracking performance with waveform library $L_{6}$ and $L_{10}$ are improved remarkably. further increasing of waveforms is not validated to error reduction and has huge computation. The design of the waveform library should be the compromise between performance and complexion.

\section{Conclusions}

The construction method of LFM waveform library for cognitive radar is investigated in this paper. The interact multiple models tracking filter's theory is elaborated. With the waveform selection criteria derived on the basis of information theory, a strategy of waveform library construction through combination of fractional Fourier transformation and chirp rate is analyzed. The simulations shows that construction of waveform library with a number of waveforms can improve the tracking performance.

\section{References}

1. Goodman $\mathrm{N}$ A, P R Venkata, M A Neifeld. "Adaptive waveform design and sequential hypothesis testing for target recognition with active sensors" [J]. IEEE Journal of Selected Topics in Signal Processing, 2007, 1(1), pp. 105-113.

2. Haykin S. "Cognitive radar: a way of the future" $[\mathrm{J}]$. IEEE Signal Processing Magazine, 2006, 23(1):3040.

3. Haykin S. "Cognitive dynamic systems" [J] IEEE IC-ASSP, 2007: 1369-1372.

4. Haykin S, Yanbo Xue and Timothy N. "Davidson Optimal Waveform Design for Cognitive Radar" [J], IEEE Asilomar, 2008, 3-7.

5. Arasaratnam I, Haykin S. "Cubature Kalman Filters" [J], IEEE Transaction on automatic control, 2009, 54(6), 1254-1269.

6. Haykin S, Amin Zia, Arasaratnam I, et al. "Cognitive tracking radar" [J], IEEE Xplore, 2010, 1467 1470.

7. $\mathrm{Lu} \mathrm{J}, \mathrm{Hu} \mathrm{W}, \mathrm{Yu}$ W. "Adaptive beam scheduling algorithm for agile beam radar in multi-target tracking” [C]. Shanghai, 2007.
8. Li Y, Sira S P, Moran B, et al. "Adaptive Sensing of Dynamic Target State in HeavySea Clutter" [C]. 2007.

9. Boers Y Driessen H, Zwaga J. "Adaptive MFR parameter control: fixed against variable probabilities of detection" [J]. IEEE ProceedingsRadar, Sonar and Navigation, 2006, 153(1): 2-6.

10. Rihaczek A W. "Radar Waveform Selection-A Simplified Approach" [J]. IEEE Transactions on Aerospace and Electronic Systems, 1971, AES-7(6): 1078-1086.

11. Cochran D, Suvorova S, Howard S D, et al. "Waveform Libraries" [J]. IEEE Signal Processing Magazine, 2009, 26(1): 12-21.

12. Savage C O, Moran B. "Waveform selection for maneuvering targets within an IMM framework" [J]. IEEE Transactions on Aerospace and Electronic Systems, 2007, 43(3): 1205-1214.

13. TAN Jiajia, ZHANG Jianqiu. "New waveform selection approach to tracking maneuver targets" [J], Systems Engineering and Electronics. 2011, 33(3): 515-522, 543.

14. Suvorova S, Howard S D, Moran W. "Waveform libraries for radar tracking applications" [J], Waveform Diversity Conference, 2004.

15. Suvorova S, Howard S D, Moran W. "Generalized Frequency Modulated Waveform Libraries for Radar Tracking Applications" [J], IEEE Asilomar, 2009.

16. Niu R, Willett P. "Tracking considerations waveform for range and range-rate measurement" [J], IEEE Trans. On Aerospace and Electronic Systems, 2002, 38(2): 467 487. 